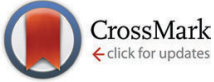

Cite this: Phys. Chem. Chem. Phys., 2015, 17, 9802

Received 21st January 2015, Accepted 27th February 2015 DOI: $10.1039 / c 5 c p 00376 h$

www.rsc.org/pccp

\title{
Modeling the local potential at Pt nanoparticles in polymer electrolyte membranes
}

\author{
Mohammad Javad Eslamibidgoli, Pierre-Éric Alix Melchy and Michael H. Eikerling*
}

\begin{abstract}
We present a physical-analytical model for the potential distribution at Pt nanodeposits in a polymer electrolyte membrane (PEM). Experimental studies have shown that solid deposits of Pt in PEM play a dual role in radical-initiated membrane degradation. Surface reactions at Pt particles could facilitate the formation as well as the scavenging of ionomer-attacking radical species. The net radical balance depends on local equilibrium conditions at Pt nanodeposits in the PEM, specifically, their equivalent local electrode potential. Our approach utilizes a continuum description of crossover fluxes of reactant gases, coupled with the kinetics of electrochemical surface reactions at Pt nanodeposits to calculate the potential distribution. The local potential is a function of the PEM structure and composition, which is determined by PEM thickness, concentrations of $\mathrm{H}_{2}$ and $\mathrm{O}_{2}$, as well as the size and density distribution of Pt particles. Model results compare well with experimental data for the potential distribution in PEMs.
\end{abstract}

\section{Introduction}

Prospects of unrivaled energy conversion efficiency and correspondingly reduced greenhouse gas emissions drive the global push towards polymer electrolyte fuel cell (PEFC) technology in automotive applications. Cost-effective utilization of materials as well as their stability and cycle life in the operating cell remain major challenges that must be successfully addressed. The durability and longevity of PEFCs are strongly affected by structural degradation of component materials, among which the polymer electrolyte membrane (PEM) is of central importance. The PEM degradation problem is critical for PEFC stacks in fuel cell buses that must operate for $>20000$ hours. $^{1}$

PEMs are exposed to thermal and mechanical stressors, coupled with the chemical attack of weak bonds of ionomer molecules by radical species, primarily ${ }^{\bullet} \mathrm{H},{ }^{\bullet} \mathrm{OH}$ and ${ }^{\bullet} \mathrm{OOH} .{ }^{2-4}$ Experimental studies linked chemical degradation of the membrane to the concentrations of these radical species. ${ }^{5,6}$

Deposits of Pt-in-the-membrane (PITM) are a consequence of Pt dissolution in the cathode catalyst layer. ${ }^{7}$ Mobile Pt ion complexes diffuse and migrate into the membrane. They form solid deposits by precipitation in the presence of $\mathrm{H}_{2}$ and $\mathrm{O}_{2}$, crossed over from anode and cathode, respectively. Solid Pt nanodeposits provide catalyst surface sites for processes involved in formation or scavenging of radicals. In addition, surface reactions at PITM could facilitate the decomposition or formation of $\mathrm{H}_{2} \mathrm{O}_{2}$. In the latter case, $\mathrm{H}_{2} \mathrm{O}_{2}$ thus formed could react at impurities such as $\mathrm{Fe}^{2+}$ to produce radicals. ${ }^{2,8}$

Department of Chemistry, Simon Fraser University, 8888 University Drive, Burnaby, BC, Canada V5A1S6. E-mail: meikerl@sfu.ca
The effect of PITM on the durability of the PEM is still under debate. Rodgers et al. have observed a strong impact of the Pt density on the degradation of Pt-impregnated membranes. ${ }^{9}$ Ohguri et al. have investigated the formation of ${ }^{\bullet} \mathrm{OH}$ at $\mathrm{Pt}$ particles in the membrane. ${ }^{10}$ In their work, ${ }^{\bullet} \mathrm{OH}$ is detected at both the anode and the cathode side but the amount is much larger for the anode side. Ghassemzadeh et al. have observed that the chemical degradation rate was higher in the presence of Pt catalyst for a $\mathrm{H}_{2}$-rich mixture of $\mathrm{H}_{2}$ and $\mathrm{O}_{2}\left(90 \% \mathrm{H}_{2}, 2 \%\right.$ $\left.\mathrm{O}_{2}, 8 \% \mathrm{Ar}\right)$ as compared to an $\mathrm{O}_{2}$-rich mixture $\left(20 \% \mathrm{O}_{2}, 2 \% \mathrm{H}_{2}\right.$, $78 \%$ Ar). ${ }^{11}$ A similar trend was reported by Aoki et al. ${ }^{12,13}$ and Ohma et al. ${ }^{14}$ Other researchers have observed severe membrane degradation in the presence of Pt particles; ${ }^{15-17}$ it has also been found that PITM can enhance durability by deactivating radicals and $\mathrm{H}_{2} \mathrm{O}_{2} \cdot{ }^{18-20}$ Conditions such as low relative humidity, high temperature, and high cell voltage accelerate chemical degradation due to an increased formation of $\mathrm{H}_{2} \mathrm{O}_{2}$ in the cell. ${ }^{21-23}$

The balance of competing processes depends on local conditions such as temperature and relative humidity as well as the structure and local chemical composition of the PEM. The local chemical composition of the PEM is determined by $\mathrm{pH}$ and by mole fractions of $\mathrm{H}_{2}$ and $\mathrm{O}_{2}$ provided at opposite membrane sides. Local values of $\mathrm{H}_{2}$ and $\mathrm{O}_{2}$ concentrations can be calculated for given transport properties, composition and thickness of the PEM. The same conditions also determine the size, shape and density distribution of Pt deposits in the PEM. ${ }^{24-27}$ On the other hand, the formation of PITM has a feedback effect on concentrations of $\mathrm{H}_{2}$ and $\mathrm{O}_{2}$ as well as membrane humidification. ${ }^{17}$ Moreover, the rates of catalytic surface processes depend on size and shape of Pt nanoparticle deposits. ${ }^{21,27-29}$ Understanding the 
impact of PITM on the chemical degradation of the PEM thus involves a complex phenomenology of local conditions and structure of the PEM, as well as structure-dependent transport and reaction processes.

The local open circuit potential (OCP) of a Pt nanoparticle, considered as a nanoelectrode in the PEM, is determined by the conditions listed above. Liu and Zuckerbrod have measured the OCP distribution at a Pt nanoprobe in the membrane, with $\mathrm{H}_{2}$ and air provided at opposite membrane sides. ${ }^{30}$ They have observed a step-like potential profile. The local electrode potential in the region close to the anode was found near $0 \mathrm{~V}$ vs. RHE. In the region near the cathode, the OCP was found to attain values between $0.8 \mathrm{~V}$ and $1.0 \mathrm{~V}$. Takaichi et al. have measured the OCP distribution determined by $\mathrm{H}_{2}$ and $\mathrm{O}_{2}$ permeation in the membrane. ${ }^{31}$ They have observed a change in the step position at different $\mathrm{O}_{2}$ partial pressure and relative humidity. Later, using the microprobe technique, Ohishi et al. have studied the distribution of the OCP. ${ }^{32}$ They have investigated the influence of operating conditions on $\mathrm{O}_{2}$ transport properties and the effect on the potential at the probe insertion position.

Understanding the problem of electrostatic potential at isolated metallic nanoparticles in an electrolyte is fundamentally interesting and of broader practical impact. Specifically, the problem of Pt nanodeposits in polymer electrolyte membranes has received significant attention in recent years. However, to the best of our knowledge only one modeling work has been devoted to this topic. ${ }^{33}$ Atrazhev et al. have developed a model to predict the potential distribution at a single $\mathrm{Pt}$ particle in the PEM. ${ }^{33}$ Their model employs an "ad hoc" formulation of the problem, in which the surface concentrations of reactant gases, $\mathrm{H}_{2}$ and $\mathrm{O}_{2}$, at a spherical Pt particle are obtained as functions of particle position. The relations are substituted in the Butler-Volmer equations and the charge balance condition is applied to numerically calculate the local mixed potential at the particle. Their model, however, suffers from physically inconsistent relations obtained for the current densities of hydrogen oxidation reaction (HOR) and oxygen reduction reaction (ORR), i.e. eqn (36) and (37) in their paper. ${ }^{33}$ As discussed in their paper, at the position of the potential step, both HOR and ORR are controlled by diffusion of reactant gases to the particle surface; thus, at this position, each of the current densities must be independent of the particle potential. However, in their relevant equations the dependency still exists. In addition, the implementation and parameterization of HOR and ORR kinetics employed in their model are incompatible with experimental data. ${ }^{34-36}$

In this paper, we present a rigorous and self-consistent formulation of the problem, which results in a physical-analytical model of the mixed-potential distribution at Pt nanodeposits in the PEM. The model employs continuum diffusion for the crossover of reactant gases, $\mathrm{H}_{2}$ and $\mathrm{O}_{2}$, coupled with local electrochemical reactions at the surface of $\mathrm{Pt}$ nanoparticles. The analytical solution gives the shapes of reactant concentrations and potential profile in terms of experimental parameters, including the relative concentration of $\mathrm{H}_{2}$ and $\mathrm{O}_{2}$ at the PEM boundaries, mass transfer coefficients, kinetic parameters of surface reactions at $\mathrm{Pt}$, and size and density of $\mathrm{Pt}$ particles in the PEM. Model outcomes are compared to experimental measurements of the OCP at Pt nanodeposits in the membrane. ${ }^{30,31}$

\section{The model}

The model incorporates a two-scale description of transport and reaction processes in the membrane, as illustrated in Fig. 1. At the macroscale, one-dimensional (1D) diffusion governs the distribution of redox species, i.e., $\mathrm{H}_{2}$ and $\mathrm{O}_{2}$, along the thickness variable $x$. Even though we do not consider the membrane embedded in the fuel cell configuration, we refer to the side at which hydrogen is supplied as the anode and the side at which oxygen is provided as the cathode. Concentrations of hydrogen and oxygen in the PEM are $c_{\mathrm{H}_{2}}(x)$ and $c_{\mathrm{O}_{2}}(x)$. The concentrations at the PEM boundaries are $c_{\mathrm{H}_{2}}^{0}$ at $x=0$ (anode side) and $c_{\mathrm{O}_{2}}^{0}$ at $x=l$ (cathode side). At the nanoscale, the hydrogen oxidation reaction (HOR), $\mathrm{H}_{2} \leftrightarrow 2 \mathrm{H}^{+}+2 \mathrm{e}^{-}$, and the oxygen reduction reaction (ORR), $\mathrm{O}_{2}+4 \mathrm{H}^{+}+4 \mathrm{e}^{-} \rightarrow 2 \mathrm{H}_{2} \mathrm{O}$ proceed at the surface of $\mathrm{Pt}$ nanoparticles, which we assume spherical. As Pt nanoparticles are not connected to an electron source/sink, the resulting OCP must fulfill a condition that the rates of HOR and ORR processes are balanced at the particle level.

As shown in Fig. 2, four regimes can be distinguished to describe the mixed reaction kinetics at the surface of Pt nanodeposits in the PEM. The distinction is based on the comparison of diffusion and kinetically-limited current densities of HOR and ORR at the spherical Pt nanoelectrode. The diffusion limited current density of the HOR is ${ }^{37}$

$$
j_{\mathrm{HOR}}^{d}=2 F c_{\mathrm{H}_{2}}(x) \frac{D_{\mathrm{H}_{2}}^{\text {nano }}}{r_{0}},
$$

and that of the ORR is

$$
j_{\mathrm{ORR}}^{\mathrm{d}}=4 F c_{\mathrm{O}_{2}}(x) \frac{D_{\mathrm{O}_{2}}^{\text {nano }}}{r_{0}},
$$

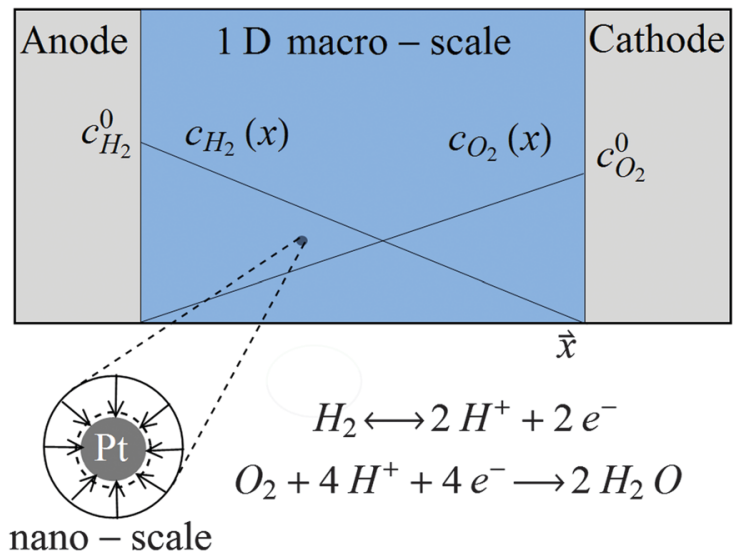

Fig. 1 Model representation of a Pt nanoparticle in the membrane. The model assumes macroscale diffusion of $\mathrm{H}_{2}$ and $\mathrm{O}_{2}$ from the opposite membrane boundaries, coupled with local diffusion around Pt nanodeposits and reactions (HOR and ORR) at the Pt surface. 


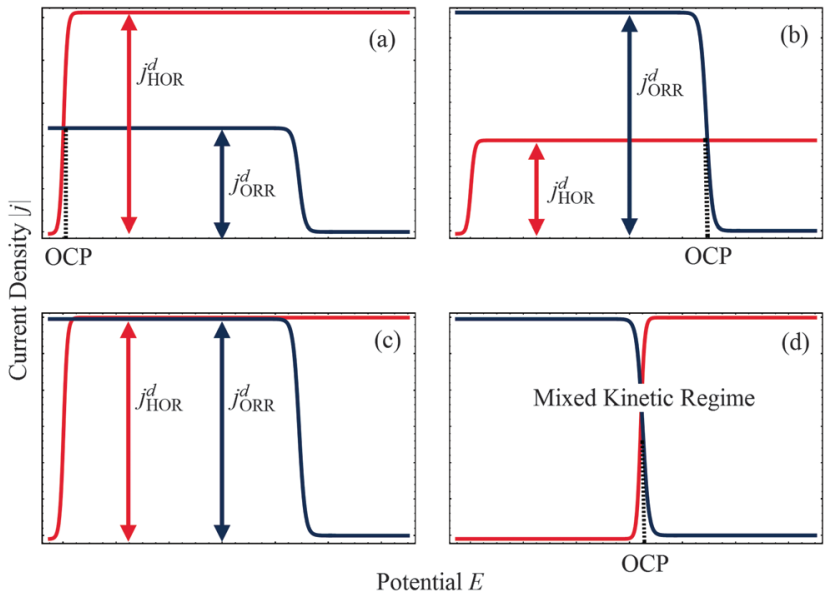

Fig. 2 Schematic representation of HOR and ORR polarization curves, indicating open circuit potential (OCP). Plots (a) and (b) correspond to mixed diffusion-kinetic regimes for HOR and ORR. For the situation depicted in (c) both reactions are limited by local diffusion, whereas in (d) both reactions are controlled by the kinetics of surface reactions.

where $F$ is the Faraday constant, $r_{0}$ is the Pt particle radius, $D_{\mathrm{H}_{2}}^{\text {nano }}$ and $D_{\mathrm{O}_{2}}^{\text {nano }}$ are the diffusion coefficients of $\mathrm{H}_{2}$ and $\mathrm{O}_{2}$ at the nanoscale, respectively.

For the faradaic current densities of HOR and ORR at the particle surface, we use expressions in the form of the ButlerVolmer equation, i.e.,

$$
j_{\mathrm{HOR}}=j_{\mathrm{HOR}}^{0}\left(\left(\frac{c_{\mathrm{H}_{2}}^{\mathrm{s}}(x)}{C_{\mathrm{ref}}}\right) \exp \left[\frac{\alpha_{\mathrm{HOR}}^{\mathrm{a}}}{b} E(x)\right]-\exp \left[-\frac{\alpha_{\mathrm{HOR}}^{\mathrm{c}}}{b} E(x)\right]\right),
$$

and

$$
\begin{aligned}
j_{\mathrm{ORR}}= & j_{\mathrm{ORR}}^{0}\left(\left(\frac{c_{\mathrm{O}_{2}}^{\mathrm{s}}(x)}{C_{\mathrm{ref}}}\right) \exp \left[-\frac{\alpha_{\mathrm{ORR}}^{\mathrm{c}}}{b}\left(E(x)-E^{\mathrm{eq}}\right)\right]\right. \\
& \left.-\exp \left[\frac{\alpha_{\mathrm{ORR}}^{\mathrm{a}}}{b}\left(E(x)-E^{\mathrm{eq}}\right)\right]\right),
\end{aligned}
$$

where $b=\frac{R T}{F}, c_{\mathrm{H}_{2}}^{\mathrm{s}}$ and $c_{\mathrm{O}_{2}}^{\mathrm{s}}$ are surface concentrations of $\mathrm{H}_{2}$ and $\mathrm{O}_{2}$, respectively, and $C_{\mathrm{Ref}}=40.88 \times 10^{-6} \mathrm{~mol} \mathrm{~cm} \mathrm{~cm}^{-3}$ is a reference concentration. ${ }^{38}$ It corresponds to the $\mathrm{O}_{2}$ concentration at standard conditions. $\alpha^{\mathrm{a}}$ and $\alpha^{\mathrm{c}}$ are the anodic and cathodic electron transfer coefficients, $j_{\text {HOR }}^{0}$ and $j_{\text {ORR }}^{0}$ are the exchange current densities of HOR and ORR, and $E^{\mathrm{eq}}$ is the equilibrium potential of ORR ( $v s$. RHE). $R$ and $T$ are the gas constant and temperature, respectively.

For a Pt nanodeposit at position $x$, one of four possible scenarios will determine the local value of the OCP, $E(x):(1)$ If $j_{\text {HOR }}^{\mathrm{d}}>j_{\text {ORR }}^{\mathrm{d}}$, then the HOR is controlled by reaction kinetics and the ORR is diffusion-limited, as shown in Fig. 2(a); in this case, $E(x)$ remains close to the equilibrium potential of the HOR. (2) If $j_{\text {HOR }}^{\mathrm{d}}<j_{\text {ORR }}^{\mathrm{d}}$, then the HOR is diffusion-limited and the ORR is kinetically controlled; in this case, $E(x)$ shifts towards the equilibrium potential of the ORR, as illustrated in Fig. 2(b). (3) The transition between the two cases occurs at the position where $j_{\mathrm{HOR}}^{\mathrm{d}}=j_{\mathrm{ORR}}^{\mathrm{d}}$, as shown in Fig. $2(\mathrm{c})$; in this regime, the current density at the particle is independent of $E(x)$; it corresponds to a step-like change in $E(x)$ at a position $x_{0}$. From the condition of equal diffusion-limited current densities in this regime, we will determine the value of $x_{0}$. (4) The last possible scenario is that both reactions are controlled kinetically, as depicted in Fig. 2(d); in this case, $E(x)$ is found from the condition $j_{\mathrm{HOR}}=j_{\mathrm{ORR}}$, using eqn (3) and (4).

Following the distinction of different kinetic regimes for the local current density at the nanoparticle surface, the PEM can be divided into two spatial regions, viz. an anodic region at $x<x_{0}$, in which $j_{\text {HOR }}^{\mathrm{d}}>j_{\text {ORR }}^{\mathrm{d}}$ and a cathodic region at $x>x_{0}$, in which $j_{\text {HOR }}^{\mathrm{d}}<j_{\text {ORR }}^{\mathrm{d}}$. In the anodic region, the HOR current is kinetically controlled, whereas the ORR is usually in the diffusion-limited regime. In the cathodic region, the ORR is kinetically controlled, whereas the HOR is usually in the diffusion-limited regime.

If a reaction at the Pt surface is kinetically controlled, the surface concentration of redox species will be equal to the bulk concentrations, i.e. $c_{\mathrm{H}_{2}}^{\mathrm{s}}=c_{\mathrm{H}_{2}}(x)$ in the anodic region and $c_{\mathrm{O}_{2}}^{\mathrm{s}}=$ $c_{\mathrm{O}_{2}}(x)$ in the cathodic membrane region. For diffusion-limited reactions, we will assume zero surface concentration of the transport-limited reactant, i.e. $c_{\mathrm{O}_{2}}^{\mathrm{s}} \approx 0$ in the anodic region and $c_{\mathrm{H}_{2}}^{\mathrm{s}} \approx 0$ in the cathodic region. As we are interested in obtaining analytical solutions for the potential profile $E(x)$, we will employ modified forms of the Butler-Volmer expressions in eqn (3) and (4), given by

$$
j_{\mathrm{HOR}}=2 j_{\mathrm{HOR}}^{0}\left(\frac{c_{\mathrm{H}_{2}}(x)}{C_{\mathrm{ref}}}\right) \sinh \left[\frac{1}{2 b} E(x)\right],
$$

and

$$
j_{\mathrm{ORR}}=j_{\mathrm{ORR}}^{0}\left(\frac{c_{\mathrm{O}_{2}}(x)}{C_{\mathrm{ref}}}\right) \exp \left[-\frac{1}{b}\left(E(x)-E^{\mathrm{eq}}\right)\right] .
$$

In eqn (5) and (6), we assume $\alpha_{\mathrm{HOR}}^{\mathrm{a}}=\alpha_{\mathrm{HOR}}^{\mathrm{c}}=\frac{1}{2}$ (ref. 34) and $\alpha_{\mathrm{ORR}}^{\mathrm{c}}=1 .^{35,36}$ In eqn (5) the sinh-expression for the faradaic current density of the HOR represents a common interpolation. ${ }^{39}$ It allows for a continuous description of the HOR rate in the limit of small overpotential, $E(x) \leq b / 3$, where $c_{\mathrm{H}_{2}}(x) \approx C_{\text {ref }}$, and high overpotential, $E(x) \geq 3 b$, where $2 \sinh [E(x) / 2 b] \approx$ $\exp [E(x) / 2 b]$. The single term BV-equation for the ORR in eqn (6) is the standard form, accounting for the irreversible kinetics of the ORR.

We apply the charge balance condition for anodic and cathodic regions in the PEM and at the transition between these regions, to obtain analytical relations for the potential profile. In the anodic region, i.e. for $x<x_{0}$, we have

$$
j_{\mathrm{HOR}}=j_{\mathrm{ORR}}^{\mathrm{d}} \text {. }
$$

Inserting eqn (2) and (5) and solving for $E(x)$ results in

$$
E(x)=2 b \sinh ^{-1}\left[\frac{1}{2} \frac{c_{\mathrm{O}_{2}}(x)}{c_{\mathrm{H}_{2}}(x)} \frac{J_{\mathrm{ORR}}^{\mathrm{d}}}{j_{\mathrm{HOR}}^{0}}\right]
$$


with

$$
J_{\mathrm{ORR}}^{\mathrm{d}}=4 F C_{\mathrm{ref}} \frac{D_{\mathrm{O}_{2}}^{\text {nano }}}{r_{0}} .
$$

Similarly, in the cathodic region of the PEM, i.e. for $x>x_{0}$, we have

$$
j_{\mathrm{ORR}}=j_{\mathrm{HOR}}^{\mathrm{d}}
$$

In this case, using eqn (1) and (6) and solving for $E(x)$, gives

$$
E(x)=E^{\mathrm{eq}}-b \ln \left[\frac{c_{\mathrm{H}_{2}}(x)}{c_{\mathrm{O}_{2}}(x)} \frac{J_{\mathrm{HOR}}^{\mathrm{d}}}{j_{\mathrm{ORR}}^{0}}\right]
$$

with

$$
J_{\mathrm{HOR}}^{\mathrm{d}}=2 F C_{\mathrm{ref}} \frac{D_{\mathrm{H}_{2}}^{\text {nano }}}{r_{0}} .
$$

At $x=x_{0}$, both the current densities of HOR and ORR are controlled by diffusion and, therefore,

$$
j_{\text {HOR }}^{\mathrm{d}}=j_{\text {ORR }}^{\mathrm{d}}
$$

which leads to a unique relation between the ratio of the bulk concentrations of the reactant gases at $x_{0}$, and the diffusion coefficients,

$$
2 c_{\mathrm{O}_{2}}\left(x_{0}\right) D_{\mathrm{O}_{2}}^{\text {nano }}=c_{\mathrm{H}_{2}}\left(x_{0}\right) D_{\mathrm{H}_{2}}^{\text {nano }} .
$$

Eqn (14) can be solved to find $x_{0}$ whence the distributions $c_{\mathrm{O}_{2}}(x)$ and $c_{\mathrm{H}_{2}}(x)$ are known.

The last possible case is when both current densities of HOR and ORR are controlled by reaction kinetics,

$$
j_{\mathrm{HOR}}=j_{\mathrm{ORR}} \text {. }
$$

In this scenario, the potential profile is obtained using eqn (5) and (6), giving,

$$
E(x)=2 b \ln \left[\frac{12^{1 / 3}+\left(\kappa(x)+\sqrt{(\kappa(x))^{2}-12}\right)^{2 / 3}}{18^{1 / 3}\left(\kappa(x)+\sqrt{(\kappa(x))^{2}-12}\right)^{1 / 3}}\right],
$$

where

$$
\kappa(x)=9\left(\frac{c_{\mathrm{O}_{2}}(x)}{c_{\mathrm{H}_{2}}(x)} \frac{j_{\mathrm{ORR}}^{0}}{j_{\mathrm{HOR}}^{0}}\right) \exp \left[\frac{E^{\mathrm{eq}}}{b}\right] .
$$

Eqn (8), (11) and (16) allow expressing the potential profile in the PEM through the distribution of concentrations, $c_{\mathrm{H}_{2}}(x)$ and $c_{\mathrm{O}_{2}}(x)$. In order to obtain the concentrations, we must solve $1 \mathrm{D}$ diffusion equations for hydrogen and oxygen in the distinct PEM regions.

We define $\xi=\frac{x}{l}$ as the dimensionless coordinate along the membrane thickness and $\xi_{0}=\frac{x_{0}}{l}$ as the dimensionless position of the potential step. In the anodic region $\left(\xi<\xi_{0}\right)$, the reaction-diffusion equation for $\mathrm{O}_{2}$ is

$$
\frac{\mathrm{d}^{2} c_{\mathrm{O}_{2}}(\xi)}{\mathrm{d} \xi^{2}}=\Lambda^{-2} c_{\mathrm{O}_{2}}(\xi),
$$

and that for $\mathrm{H}_{2}$, using eqn (7), is

$$
\frac{\mathrm{d}^{2} c_{\mathrm{H}_{2}}(\xi)}{\mathrm{d} \xi^{2}}=\frac{2 \Lambda^{-2}}{\delta^{2}} c_{\mathrm{O}_{2}}(\xi),
$$

with

$$
\delta^{2}=\frac{D_{\mathrm{H}_{2}}^{\text {macro }}}{D_{\mathrm{O}_{2}}^{\text {macro }}}
$$

and

$$
\Lambda=\frac{1}{l}\left(4 \pi r_{0} n_{\mathrm{Pt}}\left(\frac{D_{\mathrm{O}_{2}}^{\text {nano }}}{D_{\mathrm{O}_{2}}^{\text {macro }}}\right)\right)^{-1 / 2},
$$

where $n_{\mathrm{Pt}}$ is the number of Pt particles per unit PEM volume. The product $\Lambda l$ represents the dimensional reaction penetration depth of $\mathrm{O}_{2}$. In the dilute limit of the Pt particle distribution, we have $\Lambda \gg 1$, and in the dense limit $\Lambda \ll 1$.

The diffusion coefficients at nanoscale and macroscale are distinguished as the structure and effective transport resistance of the medium is different at the two scales. The macroscale diffusion coefficient corresponds to diffusion in a random composite medium, with randomly mixed resistances due to polymer and water-filled domains. Nanoscale diffusion coefficients, on the other hand, approach the values for water. Therefore, the macroscopic diffusion coefficient will be smaller than the value in water by a factor that incorporates percolation effects. We consider this value as 10 because the diffusion coefficient of $\mathrm{H}_{2}$ and $\mathrm{O}_{2}$ in water ${ }^{40}$ is one order of magnitude larger than its value in Nafion. This ratio is included in $\Lambda$ in eqn (21).

Due to the large overpotential for the ORR at the anode, we assume that $\mathrm{O}_{2}$ is completely used up at $\xi=0$. The boundary conditions of eqn (18) and (19) are given by

$$
c_{\mathrm{O}_{2}}(\xi=0)=0, \quad c_{\mathrm{O}_{2}}\left(\xi_{0}\right)=c_{\mathrm{O}_{2}}^{\xi_{0}},
$$

and

$$
c_{\mathrm{H}_{2}}(\xi=0)=c_{\mathrm{H}_{2}}^{0}, \quad c_{\mathrm{H}_{2}}\left(\xi_{0}\right)=c_{\mathrm{H}_{2}}^{\xi_{0}} \text {. }
$$

The solutions of eqn (18) and (19), subject to the boundary conditions (22) and (23), are

$$
c_{\mathrm{O}_{2}}(\xi)=c_{\mathrm{O}_{2}}^{\xi_{0}} \frac{\sinh \left(\Lambda^{-1} \xi\right)}{\sinh \left(\Lambda^{-1} \xi_{0}\right)}
$$

and

$$
c_{\mathrm{H}_{2}}(\xi)=\frac{2}{\delta^{2}} c_{\mathrm{O}_{2}}^{\xi_{0}} \frac{\sinh \left(\Lambda^{-1} \xi\right)}{\sinh \left(\Lambda^{-1} \xi_{0}\right)}+\left(c_{\mathrm{H}_{2}}^{\xi_{0}}-c_{\mathrm{H}_{2}}^{0}-\frac{2}{\delta^{2}} c_{\mathrm{O}_{2}}^{\xi_{0}}\right) \frac{\xi}{\xi_{0}}+c_{\mathrm{H}_{2}}^{0} .
$$

Similarly, in the cathodic region of the $\operatorname{PEM}\left(\xi>\xi_{0}\right)$, the reaction-diffusion equation of $\mathrm{H}_{2}$ is

$$
\frac{\mathrm{d}^{2} c_{\mathrm{H}_{2}}(\xi)}{\mathrm{d} \xi^{2}}=\Lambda^{-2} c_{\mathrm{H}_{2}}(\xi)
$$

and that of $\mathrm{O}_{2}$, using eqn (10), is

$$
\frac{\mathrm{d}^{2} c_{\mathrm{O}_{2}}(\xi)}{\mathrm{d} \xi^{2}}=\frac{\Lambda^{-2} \delta^{2}}{2} c_{\mathrm{H}_{2}}(\xi) \text {. }
$$


Here, for simplicity, we assume $\left(\frac{D_{\mathrm{O}_{2}}^{\text {nano }}}{D_{\mathrm{O}_{2}}^{\text {macro }}}\right)=\left(\frac{D_{\mathrm{H}_{2}}^{\text {nano }}}{D_{\mathrm{H}_{2}}^{\text {macro }}}\right)$, so that $\Lambda$ is the same as defined in eqn (21).

The boundary conditions are

$$
c_{\mathrm{H}_{2}}\left(\xi_{0}\right)=c_{\mathrm{H}_{2}}^{\xi_{0}}, \quad c_{\mathrm{H}_{2}}(\xi=1)=0,
$$

and

$$
c_{\mathrm{O}_{2}}\left(\xi_{0}\right)=c_{\mathrm{O}_{2}}^{\xi_{0}}, \quad c_{\mathrm{O}_{2}}(\xi=1)=c_{\mathrm{O}_{2}}^{0} .
$$

The solutions of eqn (26) and (27) are

$$
c_{\mathrm{H}_{2}}(\xi)=c_{\mathrm{H}_{2}}^{\xi_{0}} \frac{\sinh \left(\Lambda^{-1}(\xi-1)\right)}{\sinh \left(\Lambda^{-1}\left(\xi_{0}-1\right)\right)}
$$

and

$$
\begin{aligned}
c_{\mathrm{O}_{2}}(\xi)= & \frac{\delta^{2}}{2} c_{\mathrm{H}_{2}}^{\xi_{0}} \frac{\sinh \left(\Lambda^{-1}(\xi-1)\right)}{\sinh \left(\Lambda^{-1}\left(\xi_{0}-1\right)\right)} \\
& +\left(c_{\mathrm{O}_{2}}^{\xi_{0}}-c_{\mathrm{O}_{2}}^{0}-\frac{\delta^{2}}{2} c_{\mathrm{H}_{2}}^{\xi_{0}}\right) \frac{\xi-1}{\xi_{0}-1}+c_{\mathrm{O}_{2}}^{0} .
\end{aligned}
$$

To obtain the equations for $c_{\mathrm{H}_{2}}^{\xi_{0}}$ and $c_{\mathrm{O}_{2}}^{\xi_{0}}$, we apply the continuity condition at $\xi=\xi_{0}$,

$$
\begin{aligned}
& \left.\frac{\mathrm{d} c_{\mathrm{H}_{2}}(\xi)}{\mathrm{d} \xi}\right|_{\xi=\xi_{0}^{-}}=\left.\frac{\mathrm{d} c_{\mathrm{H}_{2}}(\xi)}{\mathrm{d} \xi}\right|_{\xi=\xi_{0}^{+}} ; \\
& \left.\frac{\mathrm{d} c_{\mathrm{O}_{2}}(\xi)}{\mathrm{d} \xi}\right|_{\xi=\xi_{0}^{-}}=\left.\frac{\mathrm{d} c_{\mathrm{O}_{2}}(\xi)}{\mathrm{d} \xi}\right|_{\xi=\xi_{0}^{+}} .
\end{aligned}
$$

Inserting eqn (25) and (30), we obtain

$$
\begin{aligned}
& \left(\frac{2 \Lambda^{-1} \xi_{0}}{\delta^{2}} \operatorname{coth}\left(\Lambda^{-1} \xi_{0}\right)-\frac{2}{\delta^{2}}\right) c_{\mathrm{O}_{2}}^{\xi_{0}} \\
& \quad=\left(\Lambda^{-1} \xi_{0} \operatorname{coth}\left(\Lambda^{-1}\left(\xi_{0}-1\right)\right)-1\right) c_{\mathrm{H}_{2}}^{\xi_{0}}+c_{\mathrm{H}_{2}}^{0} .
\end{aligned}
$$

Similarly, by inserting eqn (24) and (31) into eqn (32), we obtain

$$
\begin{gathered}
\left(\frac{\Lambda^{-1} \delta^{2}\left(\xi_{0}-1\right)}{2} \operatorname{coth}\left(\Lambda^{-1}\left(\xi_{0}-1\right)\right)-\frac{\delta^{2}}{2}\right) c_{\mathrm{H}_{2}}^{\xi_{0}} \\
=\left(\Lambda^{-1}\left(\xi_{0}-1\right) \operatorname{coth}\left(\Lambda^{-1} \xi_{0}\right)-1\right) c_{\mathrm{O}_{2}}^{\xi_{0}}+c_{\mathrm{O}_{2}}^{0} .
\end{gathered}
$$

Eqn (33) and (34) are solved to obtain $c_{\mathrm{H}_{2}}^{\xi_{0}}$ and $c_{\mathrm{O}_{2}}^{\xi_{0}}$ (see Appendix for the solutions). In the dilute limit of Pt particles, as $\Lambda \gg 1$, $c_{\mathrm{H}_{2}}^{\xi_{0}}=c_{\mathrm{H}_{2}}^{0}\left(1-\xi_{0}\right)$ and $c_{\mathrm{O}_{2}}^{\xi_{0}}=c_{\mathrm{O}_{2}}^{0} \xi_{0}$.

Substituting the relations for $c_{\mathrm{H}_{2}}^{\xi_{0}}$ and $c_{\mathrm{O}_{2}}^{\xi_{0}}$, obtained in the Appendix, into eqn (14) the position of the potential step is found as

$$
\xi_{0}=\frac{1}{1+\frac{2}{\delta^{2}} \frac{c_{\mathrm{O}_{2}}^{0}}{c_{\mathrm{H}_{2}}^{0}}} .
$$

It indicates that as the uniform distribution of particles is assumed, $\xi_{0}$ is independent of the size and density of particles and only depends on the relevant concentrations of reactant gases at boundaries and the ratio of diffusion coefficients. This result is independent of the particle shape. The effect of particle shape appears in the form of the diffusion limited current densities and as well in $\Lambda$. $\xi_{0}$ is obtained by applying the charge balance condition for the limiting current densities of HOR and ORR as given in eqn (13). In this equation, as the parameters affecting the particle shape are assumed to be the same for HOR and ORR, they cancel out from both sides of the equation for any particle shape. In addition, for a uniform distribution the step position is independent of $\Lambda$, hence of the particle shape.

\subsection{Model parameters}

Physical properties and constants are listed in Table 1. The adopted values for the macroscale diffusion coefficients correspond to $\mathrm{H}_{2}$ and $\mathrm{O}_{2}$ diffusion in Nafion ${ }^{\circledR} 117$, evaluated at $100 \% \mathrm{RH}$ and $60{ }^{\circ} \mathrm{C}^{41}$ Exchange current densities of the ORR and HOR have been extracted from ref. 36 and 42, respectively.

As the molar concentrations of $\mathrm{H}_{2}$ and $\mathrm{O}_{2}$ in Nafion are reasonably small for the relevant partial pressures, we use Henry's law to relate $\mathrm{H}_{2}$ and $\mathrm{O}_{2}$ concentrations at the membrane boundaries to external partial pressures,

$$
c_{\mathrm{H}_{2}}^{0}=H_{\mathrm{H}_{2}} p_{\mathrm{H}_{2}}^{0} ; \quad c_{\mathrm{O}_{2}}^{0}=H_{\mathrm{O}_{2}} p_{\mathrm{O}_{2}}^{0} .
$$

with Henry's law constants $H_{\mathrm{H}_{2}}$ and $H_{\mathrm{O}_{2}}$ in Nafion.

Table 1 Physical parameters, symbols and values

\begin{tabular}{l}
\hline Description \\
Faraday constant, $F$ \\
Gas constant, $R$ \\
Temperature, $T$ \\
ORR equilibrium potential, $E^{\text {eq }}$ \\
Reference $\mathrm{H}_{2}$ molar concentration, $C_{\text {ref }}$ \\
Reference $\mathrm{O}_{2}$ molar concentration, $C_{\text {ref }}$ \\
$\mathrm{H}_{2}$ diffusion coefficient in Nafion, $D_{\mathrm{H}_{2}}^{\text {macro }}$ \\
$\mathrm{O}_{2}$ diffusion coefficient in Nafion, $D_{\mathrm{O}_{2}}^{\text {macro }}$ \\
HOR exchange current density, $j_{\mathrm{HOR}}^{0}$ \\
ORR exchange current density, $j_{\mathrm{ORR}}^{0}$ \\
Transfer coefficients for HOR, $\alpha_{\mathrm{HOR}}^{\mathrm{a}}=\alpha_{\mathrm{HOR}}^{\mathrm{c}}$ \\
Transfer coefficient for ORR, $\alpha_{\mathrm{ORR}}^{\mathrm{c}}$
\end{tabular}

$96485 \mathrm{C} \mathrm{mol}^{-1}$

$8.314 \mathrm{~J} \mathrm{~mol}^{-1} \mathrm{~K}^{-1}$

$333 \mathrm{~K}$

$1.23 \mathrm{~V}$

$40.88 \times 10^{-6} \mathrm{~mol} \mathrm{~cm}^{-3}$

$40.88 \times 10^{-6} \mathrm{~mol} \mathrm{~cm}^{-3}$

$1.2 \times 10^{-5} \mathrm{~cm}^{2} \mathrm{~s}^{-1}$

$2.1 \times 10^{-6} \mathrm{~cm}^{2} \mathrm{~s}^{-1}$

$0.8 \times 10^{-3} \mathrm{~A} \mathrm{~cm}^{-2}$

$5.4 \times 10^{-11} \mathrm{~A} \mathrm{~cm}^{-2}$

$1 / 2$

1 
Table 2 Ranges for parameters

\begin{tabular}{ll}
\hline Description & Value \\
\hline $\mathrm{H}_{2}$ pressure at membrane|anode, $p_{\mathrm{H}_{2}}^{0}, \mathrm{kPa}$ & $21-125$ \\
$\mathrm{O}_{2}$ pressure at membrane $\mid$ cathode, $p_{\mathrm{O}_{2}}^{0}, \mathrm{kPa}$ & $21-125$ \\
Particle radius, $r_{0}, \mathrm{~nm}$ & $50-5000$ \\
Membrane thickness, $l, \mu \mathrm{m}$ & $25-200$ \\
Particle density, $n_{\mathrm{Pt}}, \mathrm{cm}^{-3}$ & $10^{7}-10^{10}$
\end{tabular}

Substituting eqn (36) into eqn (35), we obtain

$$
x_{0}=\frac{l}{1+2 \beta \frac{p_{\mathrm{O}_{2}}^{0}}{p_{\mathrm{H}_{2}}^{0}}},
$$

where

$$
\beta=\frac{D_{\mathrm{O}_{2}}^{\text {macro }} H_{\mathrm{O}_{2}}}{D_{\mathrm{H}_{2}}^{\text {macro }} H_{\mathrm{H}_{2}}} .
$$

The value of $\beta$ used in this work is taken from ref. 25. It is reported as $0.38 \pm 0.05$, evaluated experimentally in permeability tests of crossover hydrogen and oxygen at $100 \% \mathrm{RH}$ and $65{ }^{\circ} \mathrm{C} .{ }^{25}$

The evaluated ranges of parameters are listed in Table 2. The ranges correspond to values of parameters reported in the literature. As a baseline for parametric studies, we consider a membrane with $l=50 \mu \mathrm{m}$ in which particles with $r_{0}=150 \mathrm{~nm}$ are uniformly distributed with $n_{\mathrm{Pt}}=5 \times 10^{9} \mathrm{~cm}^{-3}$. The range of $n_{\mathrm{Pt}}$ is estimated based on the amount of Pt in the membrane. Results of electron microscopy-energy dispersive spectroscopy analysis have been used to quantify the amount of Pt transported from cathode to the membrane. ${ }^{25}$ This study estimates that $\approx 13 \%$ of $\mathrm{Pt}$ in the cathode is transported into the membrane following 3000 potential cycles. This amount corresponds to $\approx 0.38 \mathrm{mg} \mathrm{Pt}$, if we consider a Pt loading of $0.1 \mathrm{mg}$ $\mathrm{cm}^{-2}$ for a $5 \times 5 \mathrm{~cm}$ membrane. Equivalently, this amount results in a uniform distribution of Pt particles with $r_{0}=150 \mathrm{~nm}$ and $n_{\mathrm{Pt}} \approx 10^{10} \mathrm{~cm}^{-3}$ across a membrane with $l=50 \mu \mathrm{m}$.

\section{Results and discussion}

Fig. 3 shows the concentration profiles of $\mathrm{H}_{2}$ and $\mathrm{O}_{2}$ across the PEM and the step position, $\xi_{0}$, in dilute and dense limit of Pt particles. As shown, the position of $\xi_{0}$, given by eqn (35) (or (37)), depends on the relative concentrations of reactant gases at the membrane boundaries. The step shifts towards the cathode as $p_{\mathrm{H}_{2}}^{0}$ increases relative to $p_{\mathrm{O}_{2}}^{0}$ and vice versa. In the uniform particle distribution regime, $\xi_{0}$ is independent of $\Lambda$, as can be seen from eqn (37).

As shown in Fig. 3, in the dilute limit of Pt particles, as $\Lambda \gg 1$, the concentration profiles are linear, given by $c_{\mathrm{H}_{2}}(\xi)=$ $c_{\mathrm{H}_{2}}^{0}(1-\xi)$ and $c_{\mathrm{O}_{2}}(\xi)=c_{\mathrm{O}_{2}}^{0} \xi$ (see eqn (24), (25), (30) and (31)). As $\Lambda \rightarrow 1$ concentration profiles deviate from linear curves. According to eqn (18) and (19), nonlinearity occurs when the local rate of reactant consumption is of similar order of magnitude as the rate of reactant flux. In dense limit, as $\Lambda \ll 1$, both
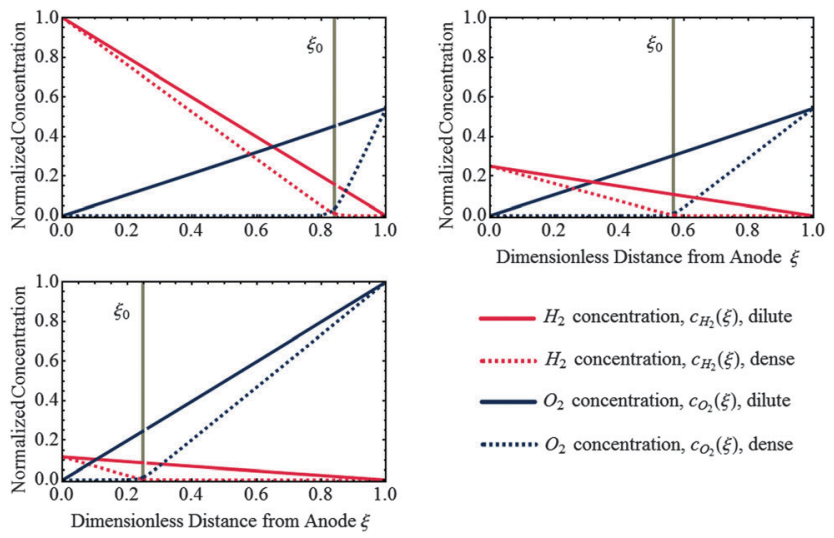

$-\mathrm{H}_{2}$ concentration, $\mathrm{C}_{\mathrm{H}_{2}}(\xi)$, dilute

.......... $\mathrm{H}_{2}$ concentration, $c_{\mathrm{H}_{2}}(\xi)$, dense

$-\mathrm{O}_{2}$ concentration, $\mathrm{c}_{\mathrm{O}_{2}}(\xi)$, dilute

........ $\mathrm{O}_{2}$ concentration, $\mathrm{c}_{2}(\xi)$, dense

Fig. 3 Analytical solution of concentration profiles of $\mathrm{H}_{2}$ and $\mathrm{O}_{2}$ in dilute limit $(\Lambda \gg 1)$ and dense limit $(\Lambda \ll 1)$ of uniformly distributed Pt deposits for various relative concentrations of $\mathrm{H}_{2}$ and $\mathrm{O}_{2}$ at the PEM boundaries. The position of potential step, $\xi_{0}$, is indicated as vertical dashed line which is independent of particle density.

concentrations approach to zero at $\xi_{0}$ which is expected as a trivial solution to eqn (14).

For the case of a dense Pt band formed in the PEM, due to the small rate of transport and high rate of consumption at the position of the dense Pt layer, the concentration of reactants must approach to zero at the band position. In this case, the potential step is found to occur in the vicinity of the band, which is in agreement with experiment. ${ }^{25}$ The particle deposition process in the membrane is due to repeated oxidation/ dissolution and reduction/deposition of the migrated Pt ions by crossover $\mathrm{O}_{2}$ and $\mathrm{H}_{2}$, respectively. ${ }^{28}$ At $x_{0}$ optimal conditions are provided for the deposition of particles to form the Pt band.

Fig. 4 shows the potential distribution at spherical $\mathrm{Pt}$ particles in the PEM for a uniform distribution with $n_{\mathrm{Pt}}=$ $10^{10} \mathrm{~cm}^{-3}$. In Fig. 4(a)-(c), the potential profile in the mixed kinetic regime (see Fig. 2(d) and eqn (16)) is compared to those of mixed "kinetic-diffusion" regimes in the two PEM regions (see Fig. 2(a) and (b), eqn (8) and (11)). The results indicate that for particles with radius smaller than $r_{0} \approx 125 \mathrm{~nm}$, the
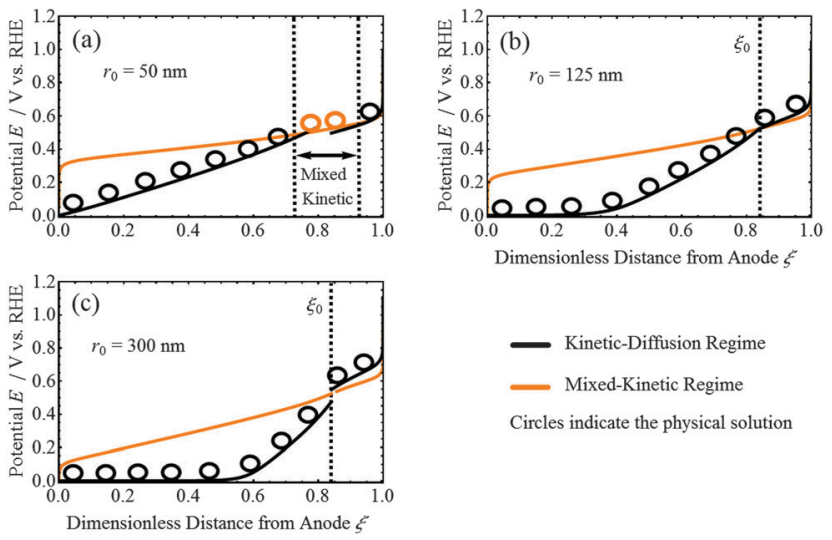

Fig. 4 Potential distribution at spherical Pt nanodeposits in the PEM. The graphs shown in (a) to (c) illustrate change in the local reaction regime with increasing size of the Pt nanoelectrode. 
transition of ORR from diffusion-limited to kinetically controlled regime occurs at $\xi<\xi_{0}$. Similarly, for this particle size range, the transition of HOR from diffusion-limited to kinetically controlled regime occurs at $\xi>\xi_{0}$. In the region between the two dashed lines in Fig. 4(a), both HOR and ORR are controlled by the reaction kinetics. This case is more relevant to Pt in the membrane, as the average PITM size reported in the literature is smaller than $125 \mathrm{~nm} .{ }^{43,44}$ It implies that in the vicinity of the potential step the concentration gradient of the reactants close to the particle surface is negligible as both reactions are kinetically controlled. On the other hand, for particles with radius greater than $r_{0} \approx 125 \mathrm{~nm}$ the transition occurs at $\xi_{0}$, as shown in Fig. 4(b) and (c) (also see Fig. 2(c) and eqn (14)).

In Fig. 5(a), the potential profile is plotted as a function of the particle size at a particle density of $n_{\mathrm{Pt}}=5 \times 10^{9} \mathrm{~cm}^{-3}$, for a membrane with thickness of $l=50 \mu \mathrm{m}$. As shown, the OCP depends on the particle size, especially close to the step. As the particle size increases the potential decreases in the anodic region and increases in the cathodic region. The OCP change as
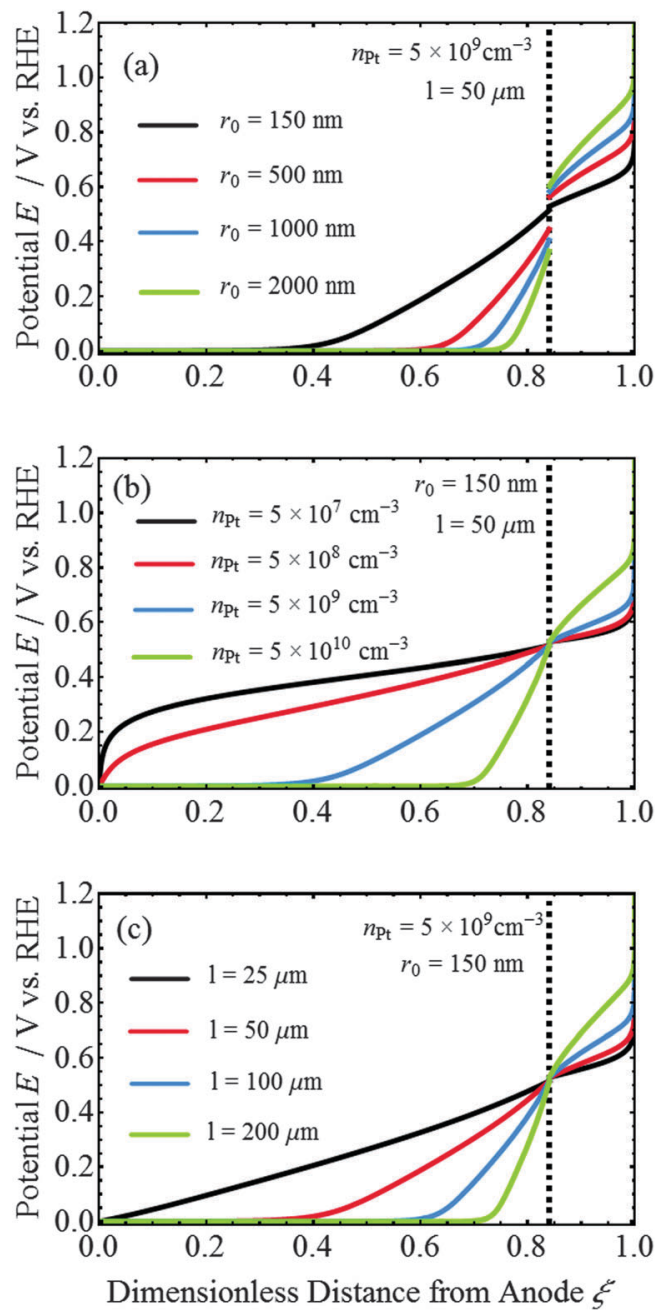

Fig. 5 Analytical solution of the potential distribution at spherical Pt nanodeposits in membrane, for (a) varying particle sizes, (b) varying particle densities, and (c) varying membrane thickness. a function of microelectrode size has been experimentally reported. ${ }^{45}$ Particle size dependence of potential has also been obtained in the model by Atrazhev et al. ${ }^{33}$

Fig. 5(b), shows the potential profile for a constant particle size (150 nm) as a function of $n_{\mathrm{Pt}}$. As $n_{\mathrm{Pt}}$ increases, the potential decreases in anodic region and increases in the cathodic region. For a constant particle size and uniform particle distribution, as the PEM thickness increases the potential decreases in the anodic region and increases in the cathodic region, as shown in Fig. 5(c). At $\xi_{0}$, the value of the potential drop only depends on the particle size. At this position, the ratio of the concentrations of reactant gases is independent of the particle density (see eqn (14)); thus, according to eqn (8) and (11) the potential drop is independent of $n_{\mathrm{Pt}}$.

Fig. 6 shows the current density distribution at Pt nanoparticles in the PEM as a function of particle density, corresponding to the potential distribution shown in Fig. 5(b). At $\xi<\xi_{0}$, the current density increases from zero to a maximum value at $\xi_{0}$ (see eqn (2)); and at $\xi>\xi_{0}$ the current density decreases to zero (see eqn (1)). As $n_{\mathrm{Pt}}$ increases, the local flux becomes smaller due to the higher consumption of crossover gases.

Fig. 7 compares the model and experimental data of the potential profile for various partial pressures of $\mathrm{H}_{2}$ and $\mathrm{O}_{2}$. In the experimental measurements of Takaichi et al.,${ }^{31}$ seven Pt microelectrode probes, with $30 \mu \mathrm{m}$ in diameter, were used to measure the OCP determined by $\mathrm{H}_{2}$ and $\mathrm{O}_{2}$ permeating in the PEM. The microelectrodes are sandwiched between eight thin membrane films, $25 \mu \mathrm{m}$ Nafion ${ }^{\circledR}$ (NRE211, Dupont, $3 \times 7 \mathrm{~cm}$ ), resulting in the total thickness of about $200 \mu \mathrm{m} . \mathrm{H}_{2}$ at ambient pressure was fed to the anode side. At the cathode side, $\mathrm{O}_{2}$ was held at a partial pressure of $21 \mathrm{kPa}, 25 \mathrm{kPa}, 101 \mathrm{kPa}$ and $119 \mathrm{kPa}$. In the experimental study performed by Liu and Zuckerbrod, $^{30}$ flattened Pt wires with an initial diameter of 25 or $50 \mu \mathrm{m}$ were used. Two Pt microelectrodes were laminated within a membrane electrode assembly with a dimension of $5 \times 5 \mathrm{~cm}$. Three layers of membranes were used. Two $10 \mu \mathrm{m}$ GORE-SELECT $^{\circledR}$ (GSM) membranes sandwiched a membrane with variable thickness, $X$. The two microelectrodes were laminated in between the outer $10 \mu \mathrm{m}$ GORE-SELECT membrane and membrane $X$, both aligned with the gas inlet region, one close to the cathode and the other close to the anode.

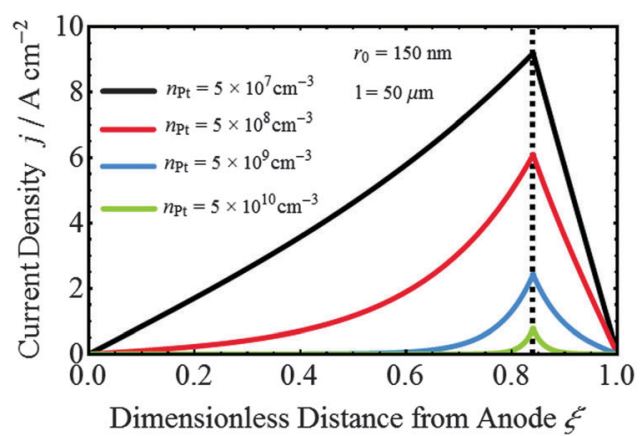

Fig. 6 Current density distributions at spherical Pt nanoelectrode calculated in the model, for various values of the particle density, corresponding to the cases depicted in Fig. 5b. 

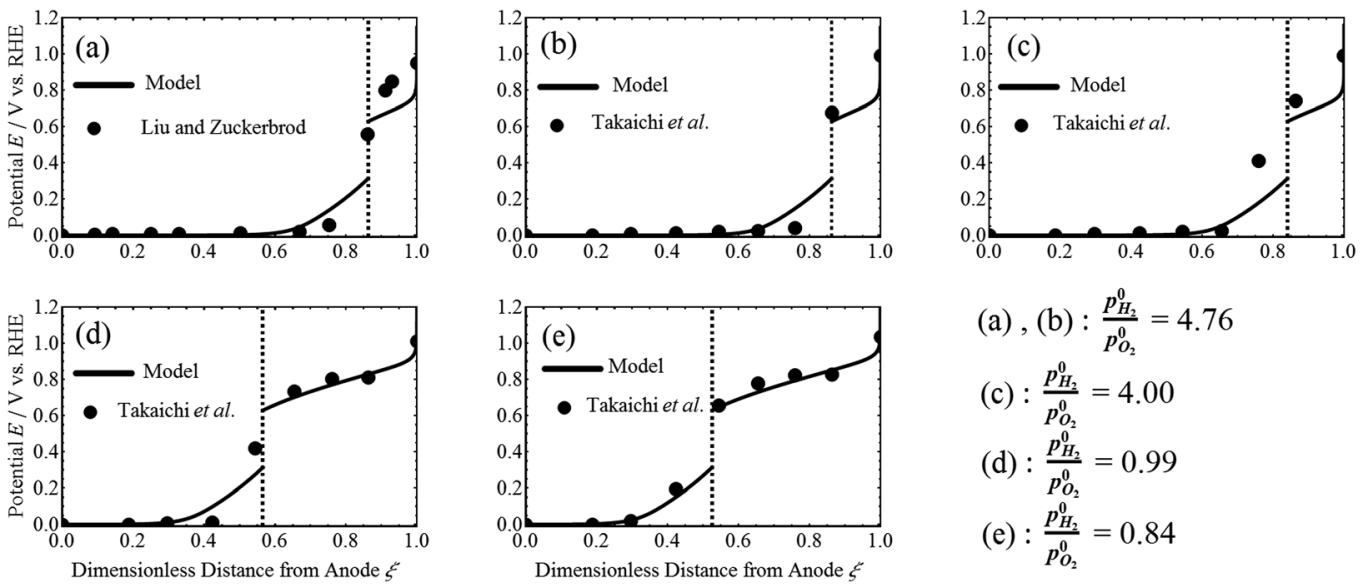
(a), (b) : $\frac{p_{H_{2}}^{0}}{p_{O_{2}}^{0}}=4.76$
(c) : $\frac{p_{H_{2}}^{0}}{p_{O}^{0}}=4.00$
(d) : $\frac{p_{H_{2}}^{0}}{p_{O_{2}}^{0}}=0.99$
(e) : $\frac{p_{H_{2}}^{\mathbf{0}}}{p_{O_{2}}^{0}}=0.84$

Fig. 7 Comparison between potential distributions and those observed in experiment ${ }^{30,31}$ for various relative partial pressures of $\mathrm{H}_{2}$ and $\mathrm{O}_{2}$ at the membrane boundaries, as indicated in the graphs. Parameters used in calculations are listed in Tables 1 and 2.

The position of the microelectrode area the cathode side was varied by changing the thickness of the membrane $X$. Both anode and cathode were standard GORE-PRIMEA ${ }^{\circledR}$ Series 5510 electrodes with $\mathrm{Pt}$ loadings of $0.4 \mathrm{mg} \mathrm{cm} \mathrm{cm}^{-2} \cdot \mathrm{H}_{2}$ and air under $100 \mathrm{kPa}$ pressure were fed to the anode and cathode sides, respectively. The potential at the microelectrodes was measured under OCP conditions at $60{ }^{\circ} \mathrm{C}$.

To compare our model with the experimental data, we assumed a spherical Pt microelectrode with $r_{0}=5 \mu \mathrm{m}$ in a membrane with $l=200 \mu \mathrm{m}$ and a dilute particle density of $n_{\mathrm{Pt}}=10^{7} \mathrm{~cm}^{-3}$. For the $\mathrm{H}_{2}$ partial pressure we assumed $100 \mathrm{kPa}$ and for the $\mathrm{O}_{2}$ partial pressure we used the values reported in experiment. The variation in microelectrode shape does not demand a modification of the model; it is merely a parametric effect in the diffusion-limited current densities at the particle surface. ${ }^{37}$ We use eqn (37) to determine $x_{0}$ with $\beta=0.38$ for all pressure settings.

The potential shapes calculated from the model are in a very good agreement with values of the potential found in experiment. As shown in Fig. 7, the step-like potential profile is very well reproduced in the model and the dependence of the position of the step to relative partial pressures at membrane boundaries is captured. Discrepancies might be due to a combination of the effect of microelectrode size and shape. Microelectrodes used in the experiments are large compared to the thickness of the membrane; hence, the spatial localization of potential values is relatively imprecise and represents effective values. In contrast, the model predicts the exact local value of the potential at a spherical microelectrode in the PEM. It is required to perform experimental studies with smaller microelectrodes in order to obtain better spatial resolution.

\section{Conclusions}

We presented an analytical model to determine the potential distribution at spherical Pt nanodeposits in the polymer electrolyte membrane. The approach incorporates a continuum description of crossover fluxes of reactant gases at the macroscale, coupled with the kinetics of electrochemical surface reactions at Pt nanodeposits. The potential distribution is obtained as a function of the local composition of the PEM, which is given by concentrations of $\mathrm{H}_{2}$ and $\mathrm{O}_{2}$ as well as the size and density distribution of Pt particles. In good agreement with experimental data, the model predicts a step-like potential profile. In the anodic region of the PEM, the potential is close to the equilibrium potential of the HOR. In the cathodic region, the potential is closer to the equilibrium potential of the ORR. For a uniform distribution of Pt particles, the position of the step is only determined by the relative partial pressures of $\mathrm{H}_{2}$ and $\mathrm{O}_{2}$ at the membrane interfaces as well as their permeation properties in the PEM. In the future we will use this model to rationalize the balance of species involved in chemical degradation of polymer electrolyte membranes.

\section{Appendix}

In this appendix, the solutions of eqn (33) and (34) for $c_{\mathrm{H}_{2}}^{\xi_{0}}$ and $c_{\mathrm{O}_{2}}^{\xi_{0}}$ are presented. Rewriting the eqn (33) and (34),

$$
\mathscr{R} c_{\mathrm{H}_{2}}^{\xi_{0}}=\mathscr{S} c_{\mathrm{O}_{2}}^{\xi_{0}}+\mathscr{T} ; \quad \mathscr{U} c_{\mathrm{H}_{2}}^{\xi_{0}}=\mathscr{V} c_{\mathrm{O}_{2}}^{\xi_{0}}+\mathscr{W}
$$

$c_{\mathrm{H}_{2}}^{\xi_{0}}$ and $c_{\mathrm{O}_{2}}^{\xi_{0}}$ are given by:

$$
c_{\mathrm{H}_{2}}^{\xi_{0}}=\frac{\mathscr{S} \mathscr{W}-\mathscr{T} \mathscr{V}}{\mathscr{U} \mathscr{S}-\mathscr{R} \mathscr{V}} ; \quad c_{\mathrm{O}_{2}}^{\xi_{0}}=\frac{\mathscr{R} \mathscr{W}-\mathscr{T} \mathscr{U}}{\mathscr{U} \mathscr{S}-\mathscr{R} \mathscr{V}} .
$$

where,

$$
\mathscr{R}=\Lambda^{-1} \xi_{0} \operatorname{coth}\left[\Lambda^{-1}\left(\xi_{0}-1\right)\right]-1,
$$

$$
\begin{gathered}
\mathscr{S}=\frac{2 \Lambda^{-1} \xi_{0}}{\delta^{2}} \operatorname{coth}\left[\Lambda^{-1} \xi_{0}\right]-\frac{2}{\delta^{2}}, \\
\mathscr{T}=-c_{\mathrm{H}_{2}}^{0},
\end{gathered}
$$




$$
\begin{gathered}
\mathscr{U}=\left[\frac{\delta^{2} \Lambda^{-1}\left(\xi_{0}-1\right)}{2} \operatorname{coth}\left[\Lambda^{-1}\left(\xi_{0}-1\right)\right]-\frac{\delta^{2}}{2}\right], \\
\mathscr{V}=\left(\xi_{0}-1\right) \Lambda^{-1} \operatorname{coth}\left[\Lambda^{-1} \xi_{0}\right]-1, \\
\mathscr{W}=c_{\mathrm{O}_{2}}^{0} .
\end{gathered}
$$

\section{Nomenclature}

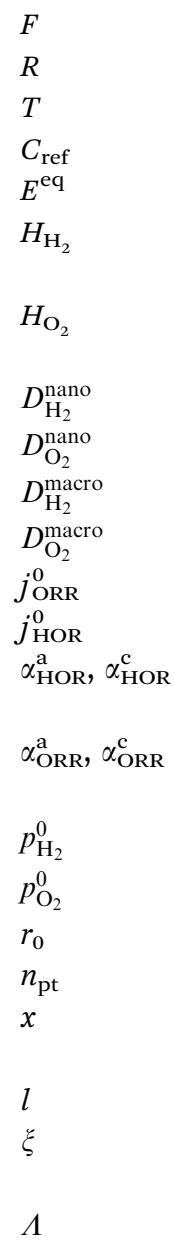

Faraday constant, $\mathrm{C} \mathrm{mol}^{-1}$

Gas constant, $\mathrm{J} \mathrm{mol}^{-1} \mathrm{~K}^{-1}$

Temperature, $\mathrm{K}$

Reference concentration, $\mathrm{mol} \mathrm{cm}^{-3}$

ORR equilibrium potential, $\mathrm{V}$

Henry's law constant for $\mathrm{H}_{2}$ in Nafion, mol cm ${ }^{-3} \mathrm{kPa}^{-1}$

Henry's law constant for $\mathrm{O}_{2}$ in Nafion, $\mathrm{mol} \mathrm{cm}{ }^{-3} \mathrm{kPa}^{-1}$

$\mathrm{H}_{2}$ diffusion coefficient at nanoscale, $\mathrm{cm}^{2} \mathrm{~s}^{-1}$

$\mathrm{O}_{2}$ diffusion coefficient at nanoscale, $\mathrm{cm}^{2} \mathrm{~s}^{-1}$

$\mathrm{H}_{2}$ diffusion coefficient at macroscale, $\mathrm{cm}^{2} \mathrm{~s}^{-1}$

$\mathrm{O}_{2}$ diffusion coefficient at macroscale, $\mathrm{cm}^{2} \mathrm{~s}^{-1}$

ORR exchange current density, $\mathrm{A} \mathrm{cm}^{-2}$

HOR exchange current density, $\mathrm{A} \mathrm{cm}^{-2}$

Anodic and cathodic transfer coefficients for HOR

Anodic and cathodic transfer coefficient for ORR

$\mathrm{H}_{2}$ pressure at membrane|anode, $\mathrm{kPa}$

$\mathrm{O}_{2}$ pressure at membrane/cathode, $\mathrm{kPa}$

Particle radius, $\mathrm{nm}$

Particle density, $\mu^{-3}$

Dimensional coordinate along PEM thickness, $\mu \mathrm{m}$

Membrane thickness, $\mu \mathrm{m}$

Dimensionless coordinate along PEM thickness, normalized by PEM thickness

Dimensionless reaction penetration depth, normalized by PEM thickness

\section{Acknowledgements}

This research is financially supported by an Automotive Partnership Canada grant and by Ballard Power Systems.

\section{References}

1 S. D. Knights, K. M. Colbow, J. St-Pierre and D. P. Wilkinson, J. Power Sources, 2004, 127, 127-134.

2 L. Gubler, S. M. Dockheer and W. H. Koppenol, J. Electrochem. Soc., 2011, 158, B755-B769.

3 X. Huang, R. Solasi, Y. Zou, M. Feshler, K. Reifsnider, D. Condit, S. Burlatsky and T. Madden, J. Polym. Sci., Part B: Polym. Phys., 2006, 44, 2346-2357.
4 V. O. Mittal, H. R. Kunz and J. M. Fenton, J. Electrochem. Soc., 2007, 154, B652-B656.

5 J. Wu, X. Z. Yuan, J. J. Martin, H. Wang, J. Zhang, J. Shen, S. Wu and W. Merida, J. Power Sources, 2008, 184, 104-119.

6 J. Healy, C. Hayden, T. Xie, K. Olson, R. Waldo, M. Brundage, H. Gasteiger and J. Abbott, Fuel Cells, 2005, 5, 302-308.

7 P. Ferreira, Y. Shao-Horn, D. Morgan, R. Makharia, S. Kocha and H. Gasteiger, et al., J. Electrochem. Soc., 2005, 152, A2256-A2271.

8 M. Ghelichi, P.-É. A. Melchy and M. H. Eikerling, J. Phys. Chem. B, 2014, 118, 11375-11386.

9 M. P. Rodgers, B. P. Pearman, L. J. Bonville, D. A. Cullen, N. Mohajeri and D. K. Slattery, J. Electrochem. Soc., 2013, 160, F1123-F1128.

10 N. Ohguri, A. Y. Nosaka and Y. Nosaka, J. Power Sources, 2010, 195, 4647-4652.

11 L. Ghassemzadeh, K.-D. Kreuer, J. Maier and K. Müller, J. Phys. Chem. C, 2010, 114, 14635-14645.

12 M. Aoki, H. Uchida and M. Watanabe, Electrochem. Commun., 2005, 7, 1434-1438.

13 M. Aoki, H. Uchida and M. Watanabe, Electrochem. Commun., 2006, 8, 1509-1513.

14 A. Ohma, S. Suga, S. Yamamoto and K. Shinohara, ECS Trans., 2006, 3, 519-529.

15 D. Zhao, B. Yi, H. Zhang and M. Liu, J. Power Sources, 2010, 195, 4606-4612.

16 N. Hasegawa, T. Asano, T. Hatanaka, M. Kawasumi and Y. Morimoto, ECS Trans., 2008, 16, 1713-1716.

17 S. Helmly, B. Ohnmacht, R. Hiesgen, E. Gülzow and K. A. Friedrich, ECS Trans., 2013, 58, 969-990.

18 N. Macauley, L. Ghassemzadeh, C. Lim, M. Watson, J. Kolodziej, M. Lauritzen, S. Holdcroft and E. Kjeang, ECS Electrochem. Lett., 2013, 2, F33-F35.

19 N. Macauley, A. S. Alavijeh, M. Watson, J. Kolodziej, M. Lauritzen, S. Knights, G. Wang and E. Kjeang, J. Electrochem. Soc., 2015, 162, F98-F107.

20 H. Hagihara, H. Uchida and M. Watanabe, Electrochim. Acta, 2006, 51, 3979-3985.

21 M. P. Rodgers, L. J. Bonville, H. R. Kunz, D. K. Slattery and J. M. Fenton, Chem. Rev., 2012, 112, 6075-6103.

22 T. Madden, D. Weiss, N. Cipollini, D. Condit, M. Gummalla, S. Burlatsky and V. Atrazhev, J. Electrochem. Soc., 2009, 156, B657-B662.

23 F. De Bruijn, V. Dam and G. Janssen, Fuel Cells, 2008, 8, 3-22.

24 V. Berejnov, Z. Martin, M. West, S. Kundu, D. Bessarabov, J. Stumper, D. Susac and A. P. Hitchcock, Phys. Chem. Chem. Phys., 2012, 14, 4835-4843.

25 W. Bi, G. E. Gray and T. F. Fuller, Electrochem. Solid-State Lett., 2007, 10, B101-B104.

26 T. Akita, A. Taniguchi, J. Maekawa, Z. Siroma, K. Tanaka, M. Kohyama and K. Yasuda, J. Power Sources, 2006, 159, 461-467.

27 K. Yasuda, A. Taniguchi, T. Akita, T. Ioroi and Z. Siroma, Phys. Chem. Chem. Phys., 2006, 8, 746-752. 
28 L. Kim, C. G. Chung, Y. W. Sung and J. S. Chung, J. Power Sources, 2008, 183, 524-532.

29 G. Diloyan, M. Sobel, K. Das and P. Hutapea, J. Power Sources, 2012, 214, 59-67.

30 W. Liu and D. Zuckerbrod, J. Electrochem. Soc., 2005, 152, A1165-A1170.

31 S. Takaichi, H. Uchida and M. Watanabe, Electrochem. Commun., 2007, 9, 1975-1979.

32 M. Ohishi, Y. Okano, Y. Ono, A. Ohma, K. Fushinobu and K. Okazaki, Int. J. Heat Mass Transfer, 2012, 55, 7213-7217.

33 V. Atrazhev, N. Erikhman and S. Burlatsky, J. Electroanal. Chem., 2007, 601, 251-259.

34 W. Sheng, H. A. Gasteiger and Y. Shao-Horn, J. Electrochem. Soc., 2010, 157, B1529-B1536.

35 D. Sepa, M. Vojnovic and A. Damjanovic, Electrochim. Acta, 1981, 26, 781-793.

36 P. D. Beattie, V. I. Basura and S. Holdcroft, J. Electroanal. Chem., 1999, 468, 180-192.
37 A. J. Bard and L. R. Faulkner, Electrochemical Methods: Fundamentals and Applications, Wiley and Sons, Hoboken, 2nd edn, 2001.

38 H. Ju, C.-Y. Wang, S. Cleghorn and U. Beuscher, J. Electrochem. Soc., 2005, 152, A1645-A1653.

39 M. M. Mench, Fuel cell engines, John Wiley \& Sons, 2008.

40 E. L. Cussler, Diffusion: mass transfer in fluid systems, Cambridge university press, 2009.

41 P. Gode, G. Lindbergh and G. Sundholm, J. Electroanal. Chem., 2002, 518, 115-122.

42 K. Neyerlin, W. Gu, J. Jorne and H. A. Gasteiger, J. Electrochem. Soc., 2007, 154, B631-B635.

43 S. Helmly, R. Hiesgen, T. Morawietz, X.-Z. Yuan, H. Wang and K. A. Friedrich, J. Electrochem. Soc., 2013, 160, F687-F697.

44 F. Ettingshausen, J. Kleemann, M. Michel, M. Quintus, H. Fuess and C. Roth, J. Power Sources, 2009, 194, 899-907.

45 J. H. Park, H. Zhou, S. J. Percival, B. Zhang, F.-R. F. Fan and A. J. Bard, Anal. Chem., 2013, 85, 964-970. 